Research Article

\title{
Theoretical Analysis of the Mechanism of the 1,3-Dipolar Cycloaddition of Benzodiazepine with N-Aryl-C-ethoxycarbonylnitrilimine
}

\author{
Khadija Nabih, Mohamed Maatallah $(\mathbb{D}$, Abdesselam Baouid, and Abdellah Jarid \\ Laboratory of Molecular Chemistry, Department of Chemistry, Faculty of Sciences Semlalia, University of Cadi Ayyad, BP. 2390, \\ Av. My Abdellah, Marrakech 40000, Morocco \\ Correspondence should be addressed to Mohamed Maatallah; m.maatallah@uca.ma
}

Received 25 September 2019; Accepted 26 December 2019; Published 27 February 2020

Academic Editor: Jolanta N. Latosinska

Copyright $(92020$ Khadija Nabih et al. This is an open access article distributed under the Creative Commons Attribution License, which permits unrestricted use, distribution, and reproduction in any medium, provided the original work is properly cited.

In this work, the mechanism and regio- and no-periselectivity of the 1,3-dipolar cycloaddition reaction of 2,4-dimethyl-3H-1,5benzodiazepine with $\mathrm{N}$-aryl-C-ethoxycarbonylnitrilimine have been studied using the DFT method at the B3LYP/6-31G(d) level of theory. IRC calculations and activation energies show that this reaction follows an asynchronous concerted mechanism. The two $\mathrm{C}=\mathrm{N}$ sites of 2,4-dimethyl-3H-1,5-benzodiazepine are easily reached by the dipole, and the energy barrier between the reagents and the transition states is too weak. The secondary barriers are traversed by the heat released in the reaction medium after the crossing of the first TS, which facilitates the addition reaction and does not require high energy. The obtained results of this study are in good agreement with experimental outcomes.

\section{Introduction}

Since the introduction of the 1,3-dipole concept established by Huisgen $[1,2]$, cycloaddition reactions have been developed considerably. In 1963, Huisgen had proposed a concerted mechanism different from the radical interpretation put forward by Firestone [3-5] and adopted for longtime. Currently, the concerted mechanism seems to be well established as a result of numerous experimental and theoretical studies [6-8]. The Huisgen cycloaddition is the reaction of a dipolarophile with a 1,3-dipolar compound that leads to 5-membered (hetero) cycles. Examples of such dipolarophiles are alkenes, alkynes, isothiocyanates, enamines, nitriles, and imines.

Over the last several decades, the extensive and detailed studies of 1,3-dipolar cycloaddition (1,3-DC) methodologies have provided organic chemists with indispensable tools to synthesize a wide array of heterocyclic products from natural products synthesis, materials science, and polymer chemistry to chemical biology $[1,6,9-27]$. For example, 1,3-dipolar cycloadditions of nitrilimines with different kinds of triple and double bonds have been extensively used in the last few decades for the synthesis of numerous five-membered $\mathrm{N}$-heterocycles of effective biological importance [28-33]. Literature offers many examples of regioselectivity of 1,3-dipolar cycloadditions (1,3-DCs) that were successfully explained using quantum chemistry- (QC-) based reactivity indices [34-41].

Although transition state (TS) theory remains the most widely used and the most rigorous approach for the study of the reactional mechanisms, the localization of TSs is not always easy. Furthermore, TS calculations are often very time-consuming when bulky substituents make parts of the studied systems.

On the other hand, the nitrilimines are characterized by their high reactivity with dipolarophiles in 1,3-dipolar cycloaddition reactions. We have attempted to prepare the nitrilimine from the corresponding precursor in the presence of triethylamine.

We performed the cycloaddition reaction of 2,4-dimethyl-3H-1,5-benzodiazepine 1 [42] with $\mathrm{N}$-aryl-Cethoxycarbonylnitrilimine 2 . This latter is, experimentally, 
generated in situ from ethyl $\mathrm{N}$-arylhydrazono-bromoglyoxylate [43] with the excess triethylamine in anhydrous benzene as solvent. It was performed at room temperature by simple agitation for 48 hours. After the usual treatment of the reaction mixture, only one product 4 was isolated (Scheme 1). Based on the experimental results [44], a first reaction mechanism is proposed.

Whatever the amount of dipole used, monocycloadduct 3 has not been isolated. On the other hand, the spectroscopic analyses show the presence of compound $\mathbf{4}$, and a double or triple bond in dipolarophiles leads, a priori, to two trans or cis isomers (Figure 1). However, the objective of the current study is to explain the mechanism of such reaction, to determine the no-periselectivity of the reaction as well as the most favored isomer, and to make a comparison with experimental data and theoretical ones obtained in this study.

\section{Computational Details}

Equilibrium geometries were optimized using the density functional theory (DFT). The computations were performed using Becke's three-parameter exchange functional with the Lee-Yang-Parr (LYP) [45-49] correlation functional and the 6-31G(d) basis set [50-52]. The Gaussian 09W [53] program series were used to perform full geometry optimizations. To check the computation level, we have performed a high-level B3LYP/6-31+G(d,p) on the reagents and the first TS and noted that the activation barrier is almost the same. So we generalized the B3LYP/6-31G(d) to localize all the extrema of the total potential surface (PES). Minima and TSs have been characterized by diagonalizing the Hessian matrix (all force constants are positive for minima and only one is negative for TSs). Moreover, intrinsic reaction coordinate (IRC) calculations were performed to check the topology of the TS environment or confirm the right (TS) connectivity to the right minima (reagents and product) $[54,55]$.

\section{Results and Discussion}

Even though the total potential energy surface (PES), without any geometrical restriction, of this $1,3-\mathrm{DC}$ reaction was meticulously explored, only two stereoisomers have been localized. These later differ of each other by both methyl groups on $\mathrm{C} 2$ and $\mathrm{C} 10$ position leading to trans or cis (Figure 1).

Thermodynamically, the results show that the formation of trans product 4 (4-trans) is energetically favored since it is lower than the cis one (4-cis) by $12 \mathrm{kcal} / \mathrm{mol}$ (Table 1). This is in perfect agreement with the experimental results [44] where only the trans one has been isolated and well characterized.

As the use of reliable ab initio methods for a reaction path treatment is too heavy for large systems, we have primarily processed our study by models. However, the 2,4dimethyl-3H-1,5-benzodiazepines $\mathbf{1}$ molecule was modeled by R1 model (Scheme 2). The total PES exploration of both approaches on $\mathrm{C}=\mathrm{N}$ bond shows that, for both cis and trans isomers, the reaction implies an unstable reactional

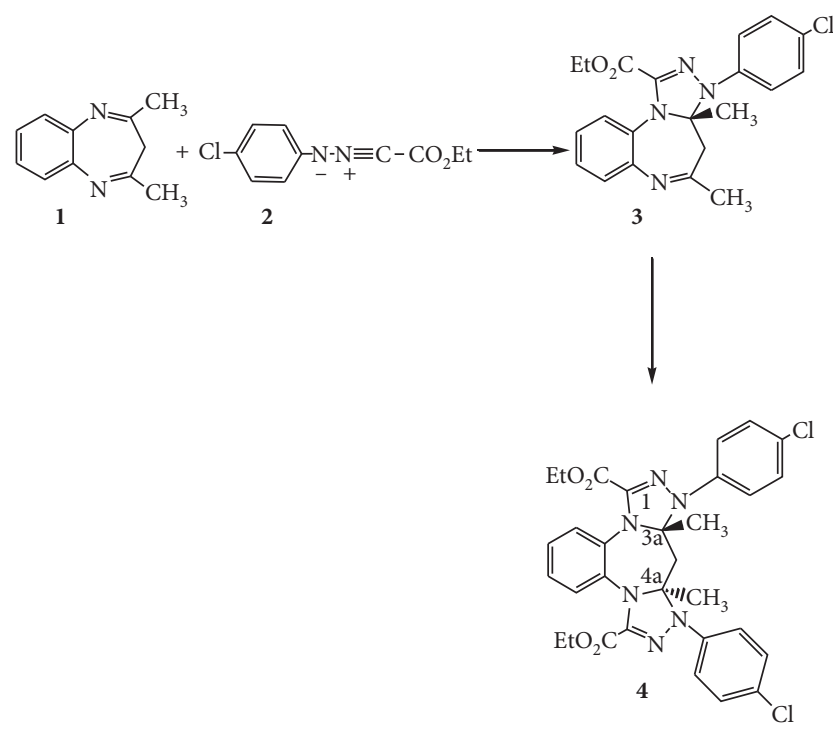

Scheme 1: Reaction of 2,4-dimethyl-3H-1,5-benzodiazepine 1 with $\mathrm{N}$-aryl-C-ethoxycarbonylnitrilimine 2 .

intermediate located between two TSs before reaching the products.

We reported in Table 2 the energetic data of all extrema (reagents, TSs (TS1 and TS2), intermediates, and products).

In approach 1, the first energy barrier TS1 is only about $3 \mathrm{Kcal} / \mathrm{mol}$ (Figure 2) above the reagent's level. The intermediate Int1 and the TS2 are so close in structure and energy that the crossing of the second barrier is done easily thanks to the released heat in the reactional medium by the transformation TS1-Int1. Figure 3 shows that the first transition corresponds to the $\mathrm{C}$ (dipole)- $\mathrm{N}$ (reagents) bond formation and the second one corresponds to the fivemembered cycle closing by the $\mathrm{N}$ (dipole)-C(reagent) bond formation. Approach 2 corresponds to the attack of the second dipole D1 on the monocycloadduct product P1. The reaction is like the first one with a first activation energy of $2 \mathrm{kcal} / \mathrm{mol}$ (Figure 2).

The vibrational normal mode and the IRC analysis of both approaches show that there are no other intermediates nor TSs on the total PES and these later correspond to the formation of the cyclic $\mathrm{C}$ (dipole)-N(reagents) and $\mathrm{N}$ (dipole)-C (reagents) bonds. The weak activation energies suggest that the addition occurs easily and seems the reason why the intermediate states Int1 and Int2 and the monocycloadduct product P1 cannot be isolated. The energetic profiles extracted from the total PES show that the reaction is exothermic, and the released energy is about $56 \mathrm{Kcal} / \mathrm{mol}$. We have depicted in Figure 3 all the optimized structures involved in the study.

Now let us analyze the mechanism on the real molecules and rationalize the regioselectivity of the cycloaddition reaction (Scheme 1). To compute the extrema corresponding to the reaction sketched in Scheme 1, we have performed DFT calculations, as detailed in the Computational Details section, on the species reported in Figure 4. This figure represents the reagents, intermediates, transition states, and products along the proposed reaction pathway leading from 


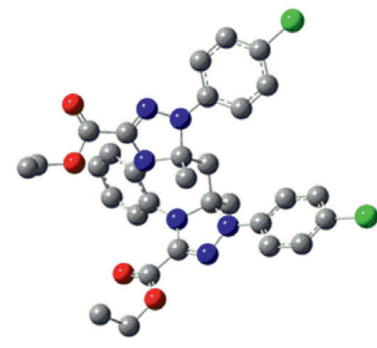

(a)

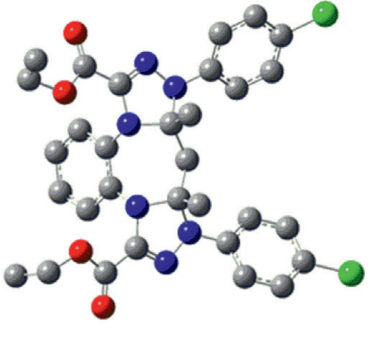

(b)

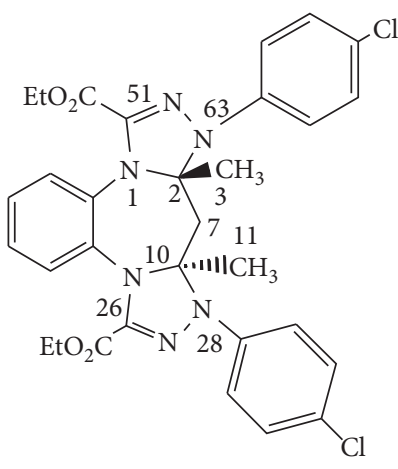

(c)

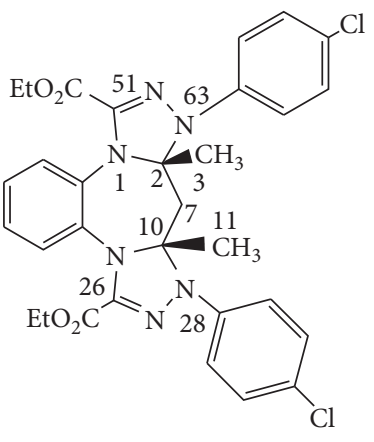

(d)

Figure 1: The lowest lying isomers (4-trans and 4-cis) on the total PES of the bis-cycloaddition. (a) 4-Trans (0.00 kcal/mol), (b) 4-cis $(12.00 \mathrm{kcal} / \mathrm{mol}),(\mathrm{c})$ 4-Trans, and (d) 4-cis.

TABLe 1: Relative energies ${ }^{\mathrm{a}}$ ( $\left.\mathrm{kcal} / \mathrm{mol}\right)$, binding lengths $(\AA)$, and dihedral angle $\left({ }^{\circ}\right)$ of the low-lying isomers of the bis-cycloaddition.

\begin{tabular}{lcc}
\hline & 4 -Trans & 4 -Cis \\
\hline$\Delta E(\mathrm{kcal} / \mathrm{mol})$ & 00 & 12 \\
$\mathrm{C} 51-\mathrm{N} 1$ & 1.413 & 1.430 \\
$\mathrm{~N} 63-\mathrm{C} 2$ & 1.502 & 1.521 \\
$\mathrm{C} 10-\mathrm{N} 28$ & 1.489 & 1.521 \\
$\mathrm{C} 26-\mathrm{N} 15$ & 1.385 & 1.429 \\
$\mathrm{C} 7-\mathrm{C} 2$ & 1.536 & 1.537 \\
$\mathrm{C} 7-\mathrm{C} 10$ & 1.552 & 1.537 \\
N63-N62 & 1.367 & 1.374 \\
N63-C64 & 1.407 & 1.420 \\
N28-N27 & 1.379 & 1.374 \\
N28-C29 & 1.397 & 1.420 \\
$\mathrm{C} 73-\mathrm{Cl} 50$ & 1.759 & 1.758 \\
$\mathrm{C} 38-\mathrm{Cl} 39$ & 1.759 & 1.758 \\
$\mathrm{C} 3-\mathrm{C} 2-\mathrm{C} 7-\mathrm{C} 10$ & 54.84 & 67.20 \\
$\mathrm{C} 11-\mathrm{C} 10-\mathrm{C} 7-\mathrm{C} 2$ & 162.13 & -67.42 \\
N63-C2-C7-C10 & 0.30 & -170.16 \\
N28-C10-C7-C2 & -70.01 & 170.00 \\
\hline
\end{tabular}

${ }^{a}$ Calculated according to 4-trans whose $E$ is -2749.107545 ua at B3LYP/6$31 \mathrm{G}(\mathrm{d})$.

1 and 2 to 4 . The energies of the TSs, TS1 and TS2, and the obtained cycloadduct 4 are reported in Table 3 and Figure 5 . In both reaction profiles of Figure 5, the starting reagents (compounds 1-D1 and 3-D1) consist of a bimolecular system corresponding to an energy minimum in the potential energy surface (PES), stabilized by weak intermolecular interactions.

Both reaction profiles involve two transition states and one intermediate. Intermediates 1-D1-Int1 and 3-D1-Int2 of both mono- and bis-1,3-DC, respectively, present only a single covalent bond between one of the two nitrogen atoms of 1 and carbon atom of $\mathbf{2}$ (Scheme 1). In detail, the first transition state of both the mono- and the bis-cycloaddition is characterized by an activation energy lower than $3 \mathrm{~kJ} / \mathrm{mol}$. The activation energy of the second transition state of the mono-cycloaddition is lower than $4 \mathrm{~kJ} / \mathrm{mol}$ for 1-D1-Int1-3 (Approach 1). The second transition state of the bis-cycloaddition has an activation energy lower than $3 \mathrm{~kJ} / \mathrm{mol}$ for 3-D1-Int2-4 (Approach 2). This value indicates that the

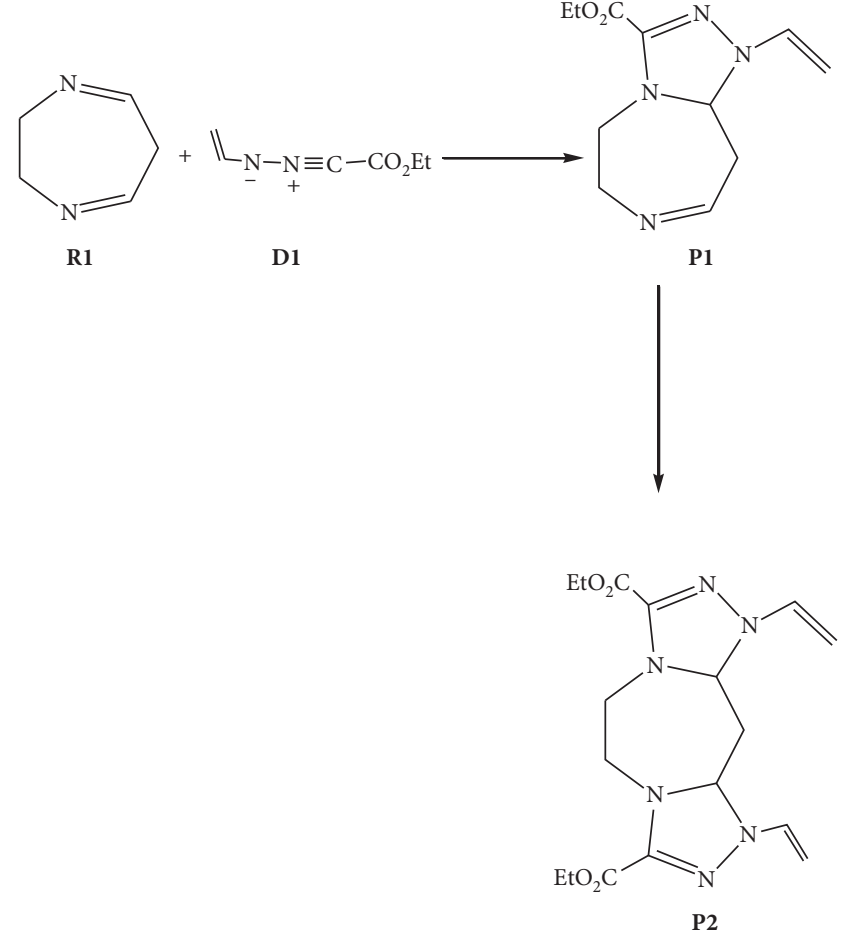

Scheme 2: Reaction of diazepine R1 with nitrilimine D1.

formation of the intermediate states 1-D1-Int1 and 3-D1Int 2 and the monocycloadduct product $\mathbf{3}$ is very easy to be obtained and cannot be isolated.

Intermediate 1-D1-Int1 can rotate its C51-N1 bond (Figure 1) to form another complex 1-D1-TS2 (Figure 4). For the second dipole attack on the second double bond, our calculations show a similar path to that of the first approach (Figure 5) with an activation energy of about $2.4 \mathrm{kcal} / \mathrm{mol}$ to cross the first TS.

Comcerning the periselectivity of the reaction, as both $\mathrm{C}=\mathrm{N}$ sites are easily reached by the dipole and the energetic barrier between reagents and TSs are too weak (less than 4 $\mathrm{Kcal} / \mathrm{mol}$ ) on one hand, and on another hand the second barriers are crossed by the heat released in the reactional medium after crossing the first TSs, making the addition 
TABle 2: Relative energies ${ }^{\mathrm{a}}(\Delta E)$ for DC reactions of reagents $\mathrm{R} 1$ and dipole D1 at B3LYP/6-31G(d).

\begin{tabular}{|c|c|c|c|}
\hline \multicolumn{2}{|c|}{ Approach 1} & \multicolumn{2}{|c|}{ Approach 2} \\
\hline & $\Delta E(\mathrm{kcal} / \mathrm{mol})$ & & $\Delta E(\mathrm{kcal} / \mathrm{mol})$ \\
\hline R1-D1 & 0.00 & P1-D1 & 0.00 \\
\hline R1-D1-TS1 & 3.00 & P1-D1-TS1 & 2.00 \\
\hline R1-D1-Int1 & -13.50 & P1-D1-Int2 & -31.00 \\
\hline R1-D1-TS2 & -13.10 & P1-D1-TS2 & -30.60 \\
\hline $\mathrm{P} 1$ & -40.20 & $\mathrm{P} 2$ & -56.30 \\
\hline
\end{tabular}

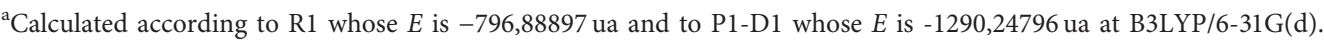
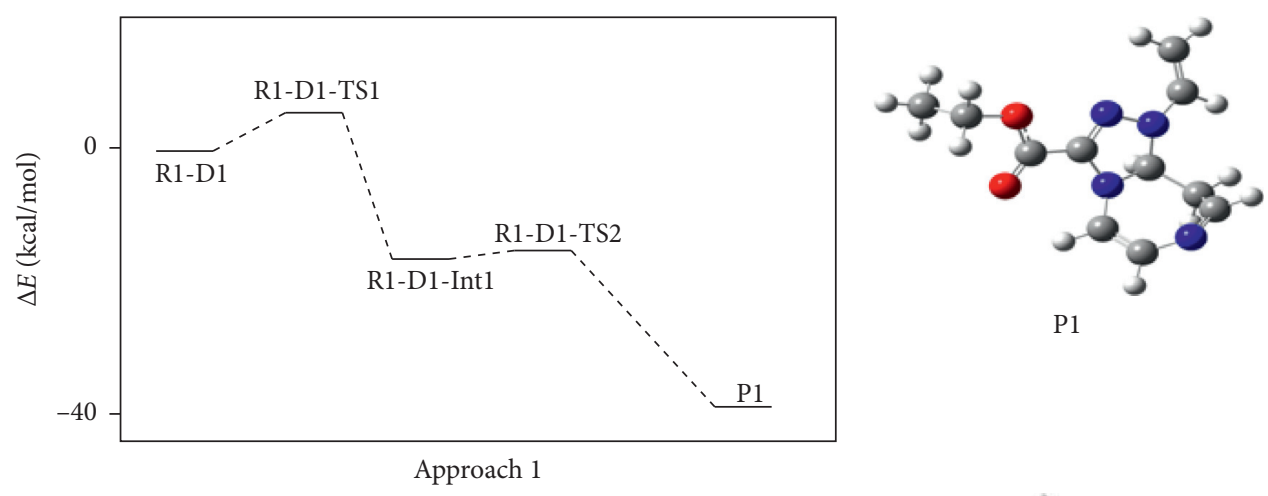

P1
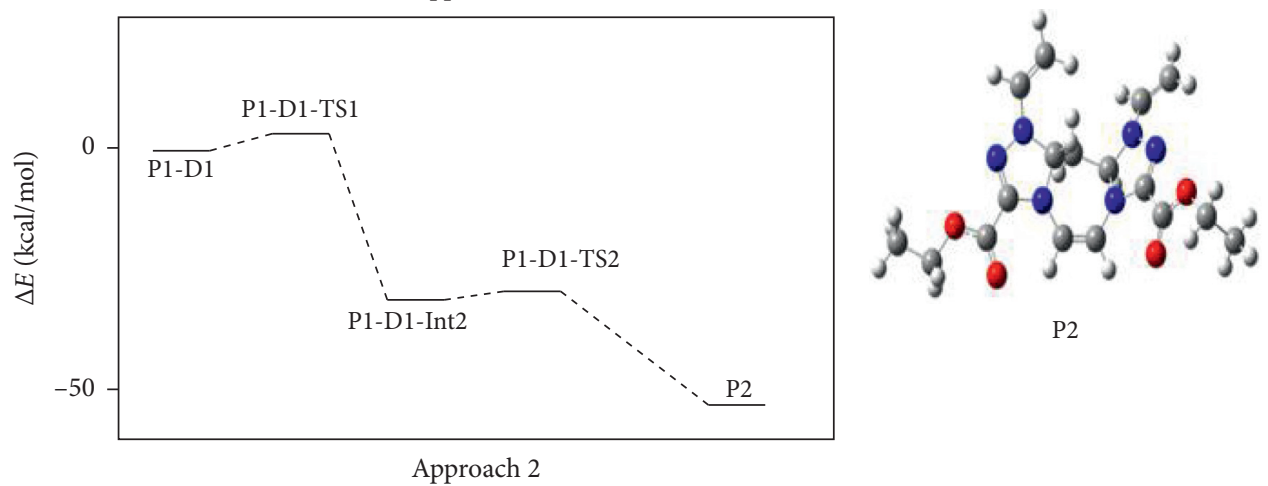

(a)

(b)

FIgURE 2: Energetic profile of both approaches at B3LYP/6-31G(d) level (a) and the lowest lying isomers of P1 and P2 products (b).<smiles>C=CN/N=C(/C(=O)OCC)N1CCC=NCC1</smiles>

R1-D1-TS1<smiles>C=CN1N=C(C(=O)OCC)N2CCNCCC12</smiles><smiles>[3H]/C(=N\NC=C)C(=O)OCC</smiles>

P1-D1-TS1<smiles>C=CN/N=C(/C(=O)OCC)N1CCC=NCC1</smiles>

R1-D1-Int1<smiles>C=CN/N=C(/OCC)N1CCC2N(C=C)N=C(C(=O)OCC)N2CC1</smiles>

P1-D1-Int2<smiles>C=CN1N=C(C(=O)OCC)N2CCN=CCC12</smiles>

R1-D1-TS2<smiles>C=CN1N=C(C(=O)OCC)N2CCN3C(C(=O)OCC)=NN(C=C)C3CC12</smiles>

P1-D1-TS2<smiles>C=CN1N=C(C(=O)OCC)N2CCN=CCC12</smiles>

P1<smiles>C=CN1N=C(C(=O)OCC)N2CCN3C(C(=O)OCC)=NN(C=C)C3CC12</smiles>

FIgURE 3: Species involved in the cycloaddition reaction (see Scheme 2 and Table 1). 


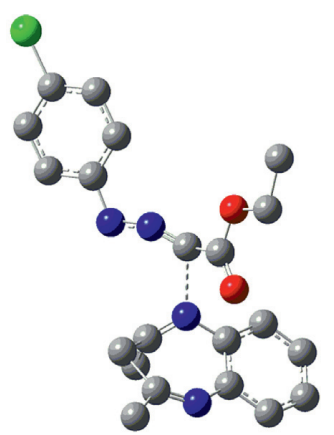

1-D1-TS1

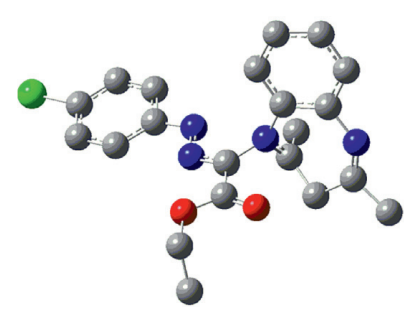

1-D1-Int1

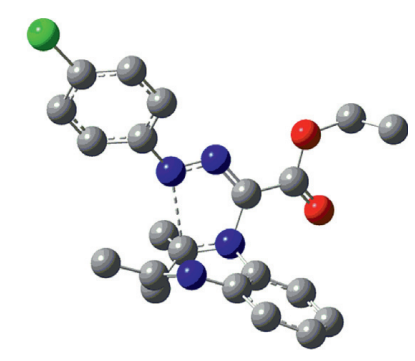

1-D1-TS2

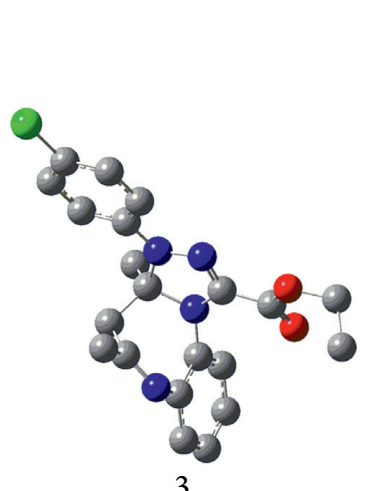

3

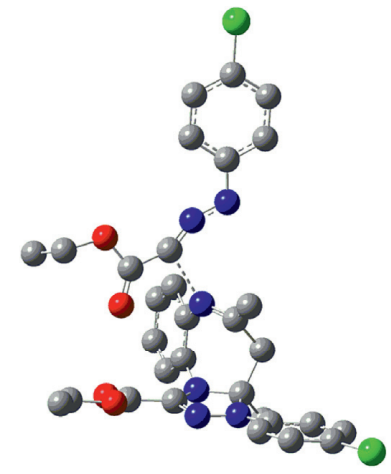

3-D1-TS1

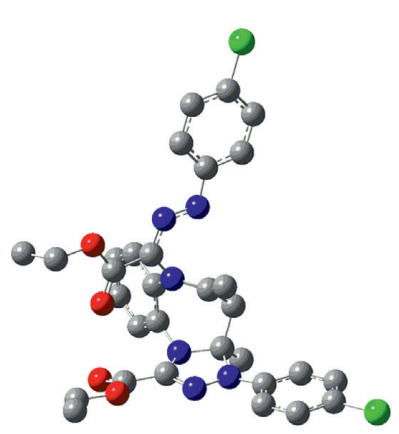

3-D1-Int2

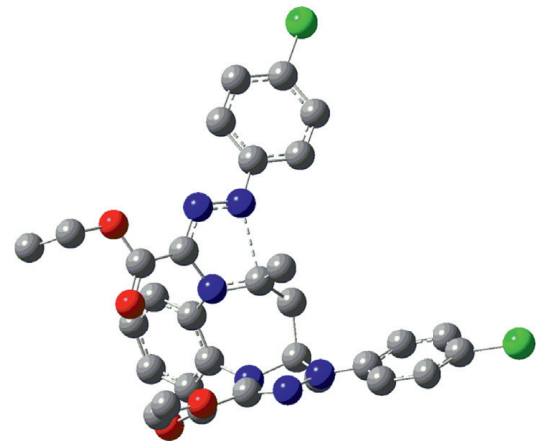

3-D1-Ts2

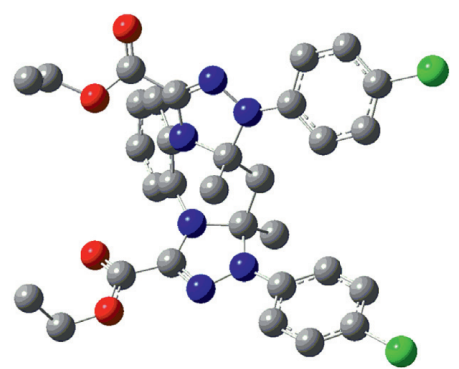

4

FIGURE 4: Selected optimized geometries of intermediates and TSs computed at B3LYP/6-31G(d) level of theory for DAC of 2,4-dimethyl$3 \mathrm{H}-1$,5-benzodiazepine 1 with $\mathrm{N}$-aryl-C-ethoxycarbonylnitrilimine 2 (hydrogen atoms have been omitted for clarity).

TABLe 3: Relative energies ${ }^{\mathrm{a}}(\Delta E)$ of the low-lying isomers for 1,3-DC of benzodiazepine 1 and dipole D1 at B3LYP/6-31G(d) level.

\begin{tabular}{lccr}
\hline & Approach 1 & & Approach 2 \\
& $\Delta E(\mathrm{kcal} / \mathrm{mol})$ & & $\Delta E(\mathrm{kcal} / \mathrm{mol})$ \\
\hline 1-D1 & 0.00 & 3-D1 & 0.00 \\
1-D1-TS1 & 1.60 & 3-D1-TS1 & 2.40 \\
1-D1-Int1 & -55.44 & 3-D1-Int2 & -20.50 \\
1-D1-TS2 & -52.17 & 3-D1-TS2 & -18.50 \\
$\mathbf{3}$ & -76.40 & $\mathbf{4}$ & -34.70 \\
\hline
\end{tabular}

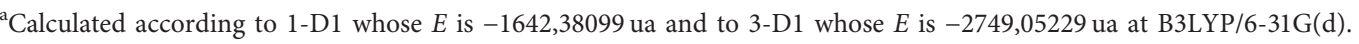

reaction easy and not requiring high energy. The second barriers are crossed by the heat released in the reactional medium after crossing the first TSs, making the addition reaction easy and not requiring high energy. This is also due to the attractive group $\mathrm{CO}_{2} \mathrm{ET}$. However, we can conclude that (i) the reaction is exothermic with a released heat of $-76,40 \mathrm{kcal} / \mathrm{mol}$ for 3 and the formation of the two biscycloaddition 4 is exothermic by $-34.70 \mathrm{~kJ} / \mathrm{mol}$; (ii) the addition on the $\mathrm{C}=\mathrm{N}$ bond is characterized by an asynchronous mechanism; (iii) the 1,3-DC of 2,4-dimethyl-3H-1,5- 


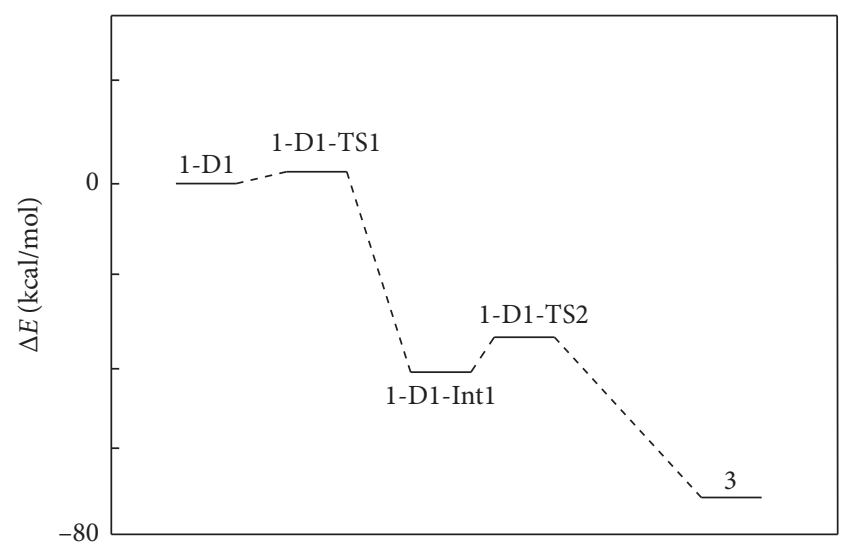

(a)

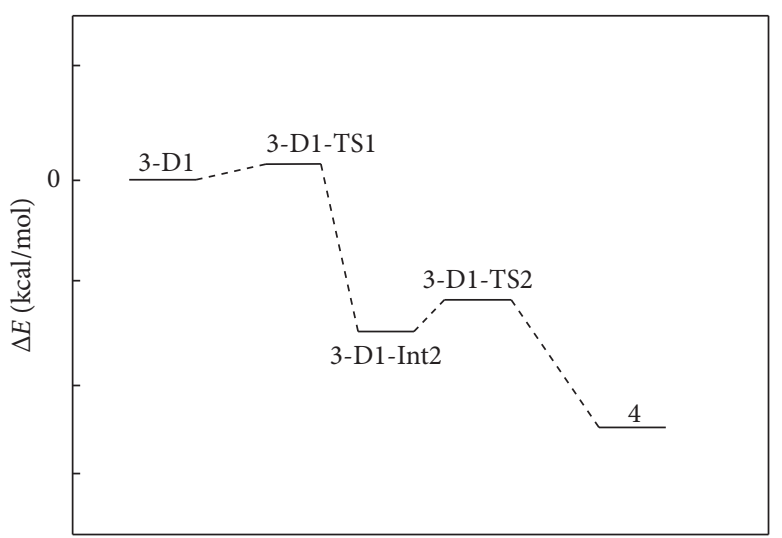

(b)

FIgURe 5: Proposed mechanisms for the DC reaction of benzodiazepine 1 and dipole D1. (a) Approach 1. (b) Approach 2.

benzodiazepine $\mathbf{1}$ and with $\mathrm{N}$-aryl-C-ethoxycarbonylnitrilimine $\mathbf{2}$ is not periselective. Finally, the energetic values obtained from this theoretical study allow us to generalize that this type of reaction is kinetically easier and thermodynamically more favorable. This is consistent with our experimental results [44].

\section{Conclusion}

The mechanism of the 1,3-dipolar cycloaddition reaction of 2,4-dimethyl-3H-1,5-benzodiazepine with $\mathrm{N}$-aryl-C-ethoxycarbonylnitrilimine was investigated using the DFT method at the $\mathrm{B} 3 \mathrm{LYP} / 6-31 \mathrm{G}(\mathrm{d})$ level of theory. The computation allowed us to conclude that

(i) The attack of the dipole on benzodiazepine $\mathbf{1}$ takes palace only in one direction, which supports that the reaction is regioselective.

(ii) The low activation energy values are consistent with the intermediate states instability since it was not observed in the spectroscopic analysis at room temperature.

(iii) The favored cycling path and the experimental regioselectivity and no-periselectivity of this cycloaddition have been rationalized by the energetic analysis. Finally, this cycloaddition follows an asynchronous mechanism.

\section{Data Availability}

No data were used to support this study.

\section{Conflicts of Interest}

The authors declare that they have no conflicts of interest.

\section{References}

[1] R. Huisgen, "1,3-dipolar cycloadditions. Past and future," Angewandte Chemie International Edition in English, vol. 2, no. 10, pp. 565-598, 1963.
[2] R. Huisgen, "Mechanism of 1,3-dipolar cycloadditions. Reply," The Journal of Organic Chemistry, vol. 33, no. 6, pp. 2291-2297, 1968.

[3] R. A. Firestone, "Mechanism of 1,3-dipolar cycloadditions," The Journal of Organic Chemistry, vol. 33, no. 6, pp. 2285-2290, 1968.

[4] R. A. Firestone, "Application of the Linnett electronic theory to organic chemistry. V. Orientation in 1,3-dipolar cycloadditions according to the diradical mechanism. Partial formal charges in the Linnett structures of the diradical intermediate," The Journal of Organic Chemistry, vol. 37, no. 13, pp. 2181-2191, 1972.

[5] R. A. Firestone, "Orientation in the 1,3-dipolar cycloaddition of diazomethane and ethyl vinyl ether," The Journal of Organic Chemistry, vol. 41, no. 12, pp. 2212-2214, 1976.

[6] R. Huisgen, "Kinetics and mechanism of 1,3-dipolr cycloadditions," Angewandte Chemie International Edition in English, vol. 2, no. 11, pp. 633-645, 1963.

[7] R. Huisgen, "1,3-dipolar cycloadditions. 76. Concerted nature of 1,3-dipolar cycloadditions and the question of diradical intermediates," The Journal of Organic Chemistry, vol. 41, no. 3, pp. 403-419, 1976.

[8] R. Huisgen, 1,3-dipolar Cycloaddition Chemistry, A. Padwa, Ed., Wiley, New York, NY, USA, 1984.

[9] L. M. Stanley and M. P. Sibi, "Enantioselective copper-catalyzed 1,3-dipolar cycloadditions," Chemical Reviews, vol. 108, no. 8, pp. 2887-2902, 2008.

[10] T. Hashimoto and K. Maruoka, "Correction to "recent advances of catalytic asymmetric 1,3-dipolar cycloadditions"," Chemical Reviews, vol. 115, no. 17, p. 9653, 2015.

[11] K. V. Gothelf and K. A. Jørgensen, "Asymmetric 1,3-dipolar cycloaddition reactions," Chemical Reviews, vol. 98, no. 2, pp. 863-910, 1998.

[12] S. Kanemasa, "Metal-assisted stereocontrol of 1,3-dipolar cycloaddition reactions," Synlett, vol. 2002, no. 9, pp. 1371-1387, 2002.

[13] S. Kanemasa, "Cornerstone works for catalytic 1,3-dipolar cycloaddition reactions," Heterocycles, vol. 82, no. 1, pp. 87-200, 2010.

[14] J. Adrio and J. C. Carretero, "Recent advances in the catalytic asymmetric 1,3-dipolar cycloaddition of azomethine ylides," Chemical Communications, vol. 50, no. 83, pp. 12434-12446, 2014.

[15] W. S. Jen, J. J. M. Wiener, and D. W. C. MacMillan, "New strategies for organic catalysis: the first enantioselective 
organocatalytic 1,3-dipolar cycloaddition," Journal of the American Chemical Society, vol. 122, no. 40, pp. 9874-9875, 2000.

[16] W. Chen, W. Du, Y.-Z. Duan, Y. Wu, S.-Y. Yang, and Y.-C. Chen, "Enantioselective 1,3-dipolar cycloaddition of cyclic enones catalyzed by multifunctional primary amines: beneficial effects of hydrogen bonding," Angewandte Chemie International Edition, vol. 46, no. 40, pp. 7667-7670, 2007.

[17] J. L. Vicario, S. Reboredo, D. Badía, and L. Carrillo, "Organocatalytic enantioselective [3+2] cycloaddition of azomethine ylides and $\alpha, \beta$-unsaturated aldehydes," Angewandte Chemie International Edition, vol. 46, no. 27, pp. 5168-5170, 2007.

[18] J. M. Baskin, J. A. Prescher, S. T. Laughlin et al., "Copper-free click chemistry for dynamic in vivo imaging," Proceedings of the National Academy of Sciences, vol. 104, no. 43, pp. 16793-16797, 2007.

[19] C. Nájera, M. de Gracia Retamosa, J. M. Sansano, A. D. Cózar, and F. P. Cossío, "Enantioselective synthesis of polysubstituted prolines by Binap-silver-catalyzed 1,3-dipolar cycloadditions," Tetrahedron: Asymmetry, vol. 19, no. 24, pp. 2913-2923, 2008.

[20] C. Nájera, M. D. G. Retamosa, and J. M. Sansano, "Catalytic enantioselective 1,3-dipolar cycloaddition reactions of azomethine ylides and alkenes by using phosphoramidite-silver(I) complexes," Angewandte Chemie International Edition, vol. 47, no. 32, pp. 6055-6058, 2008.

[21] G. Pandey, P. Banerjee, and S. R. Gadre, "Construction of enantiopure pyrrolidine ring system via asymmetric [3+2]Cycloaddition of azomethine ylides," Chemical Reviews, vol. 106, no. 11, pp. 4484-4517, 2006.

[22] R. Narayan, M. Potowski, Z.-J. Jia, A. P. Antonchick, and H. Waldmann, "Catalytic enantioselective 1,3-dipolar cycloadditions of azomethine ylides for biology-oriented synthesis," Accounts of Chemical Research, vol. 47, no. 4, pp. 1296-1310, 2014.

[23] G. Delaittre, N. K. Guimard, and C. Barner-Kowollik, "Сycloadditions in modern polymer chemistry," Accounts of Chemical Research, vol. 48, no. 5, pp. 1296-1307, 2015.

[24] E. M. Sletten and C. R. Bertozzi, "From mechanism to mouse: a tale of two bioorthogonal reactions," Accounts of Chemical Research, vol. 44, no. 9, pp. 666-676, 2011.

[25] M. Quintana, E. Vazquez, and M. Prato, "Organic functionalization of graphene in dispersions," Accounts of Chemical Research, vol. 46, no. 1, pp. 138-148, 2013.

[26] S. Luleburgaz, G. Hizal, H. Durmaz, and U. Tunca, "Modification of electron deficient polyester via Huisgen/Passerini sequence," Polymer, vol. 127, pp. 45-51, 2017.

[27] A. V. Zuraev, Y. V. Grigoriev, V. A. Budevich, and O. A. Ivashkevich, "Copper-polymer nanocomposite: an efficient catalyst for green Huisgen click synthesis," Tetrahedron Letters, vol. 59, no. 16, pp. 1583-1586, 2018.

[28] P. Caramella and P. Grtinanger, 1,3-Dipolar Cycloaddition Chemistry, A. Padwa, Ed., New York, NY, USA, vol. 1, 1984, https://trove.nla.gov.au/version/45653050.

[29] P. K. Claus, Methoden der organischenChemie (HoubenWeyl), D. Klamann, H. Hagemann, and T. Verlag, Eds., Thieme Medical Publishers, Stuttgart, Germany, 1990.

[30] A. S. Shawali, "Reactions of heterocyclic compounds with nitrilimines and their precursors," Chemical Reviews, vol. 93, no. 8, pp. 2731-2777, 1993.

[31] A. S. Shawali and C. Párkanyi, "Hydrazidoyl Halides in the synthesis of heterocycles," Journal of Heterocyclic Chemistry, vol. 17, no. 5, pp. 833-854, 1980.
[32] D. J. Minick and A. S. Shawali, "Reactions of hydrazidoyl halides with sulfur compounds," Heterocycles, vol. 20, no. 11, pp. 2239-2285, 1983.

[33] T. L. Gilchrist and R. C. Storr, Organic Reactions and Orbital Symmetry, Cambridge University Press, Cambridge, UK, 2nd edition, 1979 .

[34] P. Geerlings, F. De Proft, and W. Langenaeker, "Conceptual density functional theory," Chemical Reviews, vol. 103, no. 5, pp. 1793-1874, 2003.

[35] A. Ponti, "DFT-based regioselectivity criteria for cycloaddition reactions," The Journal of Physical Chemistry A, vol. 104, no. 39, pp. 8843-8846, 2000.

[36] G. Molteni and A. Ponti, "Arylazide cycloaddition to methyl propiolate: DFT-based quantitative prediction of regioselectivity," Chemistry - A European Journal, vol. 9, no. 12, pp. 2770-2774, 2003.

[37] A. Ponti and G. Molteni, "DFT-HSAB prediction of regioselectivity in 1,3-dipolar cycloadditions: behavior of (4substituted)benzonitrile oxides towards methyl propiolate," Chemistry-A European Journal, vol. 12, no. 4, pp. 1156-1161, 2006.

[38] A. Ponti and G. Molteni, "DFT-based quantitative prediction of regioselectivity: cycloaddition of nitrilimines to methyl propiolate," The Journal of Organic Chemistry, vol. 66, no. 15, pp. 5252-5255, 2001.

[39] G. Molteni and A. Ponti, "Assessment of mechanistic hypotheses of 1,3-dipolar cycloaddition of (arylsulfonyl)allene to nitrilimines by DFT reactivity indices," Tetrahedron, vol. 59, no. 28, pp. 5225-5229, 2003.

[40] G. Molteni and A. Ponti, "The nitrilimine-alkene cycloaddition regioselectivity rationalized by density functional theory reactivity indices," Molecules, vol. 22, no. 2, pp. 202213,2017

[41] S.-J. Li and D.-C. Fang, "A DFT kinetic study on 1,3-dipolar cycloaddition reactions in solution," Physical Chemistry Chemical Physics, vol. 18, no. 44, pp. 30815-30823, 2016.

[42] J. A. Barltrop, C. G. Richards, D. M. Russell, and G. Ryback, "225. seven-membered heterocyclic compounds. Part I. 1:5benzodiazepines and derivatives of 3:6-diaza-4:5-benzotropone," Journal of the Chemical Society (Resumed), pp. 1132-1142, 1959.

[43] D. B. Sharp and C. S. Hamilton, "Derivatives of 1,2,4-triazole and of pyrazole," Journal of the American Chemical Society, vol. 68, no. 4, pp. 588-591, 1946.

[44] K. Nabih, A. Baouid, A. Hasnaoui, M. Selkti, and P. Compain, "1,3-dipolar cycloaddition of nitrilimines to 2,4-disubstituted-3H-1,5-benzodiazepines: remarkable effect of C4-substituent on diastereoselectivity," New Journal of Chemistry, vol. 27, no. 11, pp. 1644-1648, 2003.

[45] A. D. Becke, "Density-functional exchange-energy approximation with correct asymptotic behavior," Physical Review A, vol. 38, no. 6, pp. 3098-3100, 1988.

[46] A. D. Becke, "Density-functional thermochemistry. III. The role of exact exchange," The Journal of Chemical Physics, vol. 98, no. 7, pp. 5648-5652, 1993.

[47] C. Lee, W. Yang, and R. G. Parr, "Development of the ColleSalvetti correlation-energy formula into a functional of the electron density," Physical Review B, vol. 37, no. 2, pp. 785789, 1988.

[48] P. J. Stephens, F. J. Devlin, C. F. Chabalowski, and M. J. Frisch, "Ab initio calculation of vibrational absorption and circular dichroism spectra using density functional force fields," The Journal of Physical Chemistry, vol. 98, no. 45, pp. 1162311627, 1994. 
[49] R. H. Hertwig and W. Koch, "On the parameterization of the local correlation functional. What is Becke-3-LYP?," Chemical Physics Letters, vol. 268, no. 5-6, pp. 345-351, 1997.

[50] W. J. Hehre, R. Ditchfield, and J. A. Pople, "Self-consistent molecular orbital methods. XII. Further extensions of Gaussian-type basis sets for use in molecular orbital studies of organic molecules," The Journal of Chemical Physics, vol. 56, no. 5, pp. 2257-2261, 1972.

[51] J. D. Dill and J. A. Pople, "Self-consistent molecular orbital methods. XV. extended Gaussian-type basis sets for lithium, beryllium, and boron," The Journal of Chemical Physics, vol. 62, no. 7, pp. 2921-2923, 1975.

[52] M. M. Francl, W. J. Pietro, W. J. Hehre et al., "Self-consistent molecular orbital methods. XXIII. A polarization-type basis set for second-row elements," The Journal of Chemical Physics, vol. 77, no. 7, pp. 3654-3665, 1982.

[53] M. J. Frisch, G. W. Trucks, H. B. Schlegel et al., Gaussian 09, Revision B.01, Gaussian Inc., Wallingford, CT, USA, 2009.

[54] K. Fukui, "Formulation of the reaction coordinate," The Journal of Physical Chemistry, vol. 74, no. 23, pp. 4161-4163, 1970.

[55] K. Fukui, "The path of chemical reactions-the IRC approach," Accounts of Chemical Research, vol. 14, no. 12, pp. 363-368, 1981. 